\title{
Laser Direct Writing of Copper Circuits on Flexible Substrates for Electronic Devices
}

\author{
Shi Bai ${ }^{1}$, Weiping Zhou ${ }^{1}$, Tingxiu Hou ${ }^{1}$ and Anming $\mathrm{Hu}^{1,2, *}$ \\ ${ }^{1}$ Institute of Laser Engineering; Beijing University of Technology; 100 Pingle Yuan; Beijing; 100124; PR China \\ ${ }^{2}$ Department of Mechanical, Aerospace and Biomedical Engineering, University of Tennessee \\ Knoxville, 1512 Middle Drive, Knoxville, TN 37996, USA \\ *E-mail: anminghu@bjut.edu.cn;
}

\begin{abstract}
There are increasing interests in flexible electronic devices for sports, entertainment, health monitoring and medical cares. In particular, flexible sensors (wearable strain senor or robotic controllable sensors) attached on clothing or human skins could be used as a motion detector. Copper has been considered as a potential alternative material for other expensive noble metals. By laser-induced direct reduction and transfer printing, microsensors based on conducting copper circuits was fabricated on polydimethylsiloxane or polyimide as cheap and sensitive devices. A specific electrical resistivity of $240 \mu \Omega \cdot \mathrm{cm}$ of Cu circuit was successfully achieved in an electrode of about $40 \mu \mathrm{m}$ in thickness. We demonstrate a motion sensing based on laser-reduced copper circuits with a high sensitivity and mechanical robustness.
\end{abstract}

DOI: 10.2961/jlmn.2016.03.0009

Keywords: laser direct writing, laser reduction, copper circuit, flexible device, flexible sensor

\section{Introduction}

Recently, flexible devices have been widely researched and showed remarkably promising in wearable monitoring technology [1], entertainment [2], medical cares [3] and electronic devices [4]. Some of them have entered into our daily life and contributed to improve human living quality. These flexible devices usually based on high conductive materials, such as silver, gold, conducting carbon black and/or graphene. After printing conductive materials on flexible substrates (photographic paper, polyimide or other elastomer), multi-functional sensors are fabricated for flexible electronic devices. For example, silver nanoparticle and nanowire are preferred choices used for conductive networks which tolerate strain or bending. Lee et al. proposed a new type of stretchable strain sensor fabricated on the polydimethylsiloxane (PDMS) stamp by direct transfer using silver nanoparticle [5]. The fabricated stretchable strain sensor showed highly sensitive and durable sensing performances for detecting multiple human motions. Amjadi et al. reported highly flexible, stretchable and sensitive strain sensors based on the nanocomposite of a silver nanowire network embedded in a PDMS elastomer [6]. These nanocomposite-based strain sensors showed a strong resistivity variation as a function of mechanical deformation in real-time motion detection of fingers. Both works open up applications in flexible, stretchable and wearable electronics for avatar control in the virtual environment.

However, because the prices of noble metal materials, such as silver, gold and platinum, have increased in recent years, the price of noble metal nanoparticles is pushed up. Therefore, copper has been considered as a potential replacement material for these expensive noble metals. Up to now, various photonic techniques have been developed to synthesize and sinter copper material, such as laser direct writing [7] and intense pulsed light [8]. Besides, copper oxide is attractive as copper source to synthesize copper nanoparticles, because the copper oxide nanoparticle is much more stable than a copper nanoparticle in ambient conditions. However, photonic reduction level of copper oxide is usually limited due to light screening inside the agglomerate copper nanoparticles [7, 9]. To overcome this barrier, we developed a new laser reduction method in which copper salt is employed to replace copper oxide.

In this study, we displayed that element copper could be formed from copper salt by laser reduction and conductive copper circuits were fabricated by laser direct writing. After transferred onto PDMS, the flexible sensor based on conductive copper circuits showed high sensitivity and mechanical robustness. This work led to a pathway towards a practical production of conductive copper flexible films

\section{Experiment}

\subsection{Copper salt preparation}

To prepare $\mathrm{Cu}$ salt, $2 \mathrm{ml}$ ethylene glycol (Tianjin FuChen) was poured into a beaker and $2.5 \mathrm{~g}$ copper (II) nitrate trihydrate $\left(\mathrm{CuNO}_{3} \cdot 3 \mathrm{H}_{2} \mathrm{O}\right)$ (Tianjin FuChen) was added. The solution was stirred at least 20 min making sure $\mathrm{CuNO}_{3}$ was dissolved. The liquid was heated to $100{ }^{\circ} \mathrm{C}$ for $10 \mathrm{~min}$, then the solution turned into a black green color. After cooled down to room temperature, $50 \mu \mathrm{l}$ formic acid (Tianjin FuChen) was added into the solution.

\subsection{Laser reduction and transfer printing}

Before coating, the surface of substrate was treated by reaction ion etching (RIE) for 1min 30s under the condition of $\mathrm{O}_{2} 0.2 \mathrm{~L} / \mathrm{min}$, 200W to enhance the substrate hydrophilicity. The spinning rate was $700 \mathrm{rpm}$ about $10 \mathrm{~s}$ for coating 
copper salt. To write the copper circuit, a continuous wave diode laser (K808DAECN-30.00W, BWT Beijing LTD.) with a wavelength $808 \mathrm{~nm}$ was used. The laser beam spot with about $150 \mu$ m diameter $\left(1 / \mathrm{e}^{2}\right)$ was focused by a microscope objective (NA=0.3). The scanning speed was $5 \mathrm{~mm} / \mathrm{s}$. Figure 1 (a) shows the configuration of the laser direct reduction system. After laser processing, the specimen was cleaned by DI water in order to wipe off the residual copper salt. For patterning on PDMS, the first step is to mix a PDMS pre-polymer and a curing agent (sylgard 184, Dow corning, USA) with a weight ratio of 10: 1 . After all bubbles ran off, the mixture was poured over the glass slide. After further curing in a drying oven at $80^{\circ} \mathrm{C}$ for $4 \mathrm{~h}$, the copper attached PDMS tape with a thickness of $3 \mathrm{~mm}$ was detached from the glass as shown in figure 1 (b)

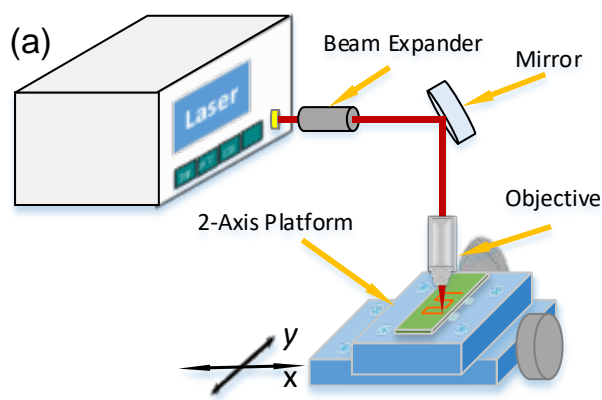

(b)

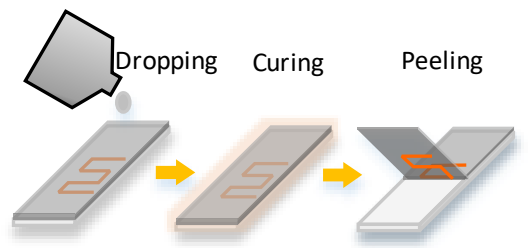

Fig 1. (a) Schematic of the experimental setup for laser direct reduction. (b) Schematic illustration of fabrication of flexible sensor based on copper circuit.

\subsection{Characterization}

High-resolution images of the Cu patterns were observed by field emission scanning electron microscopy (Hitachi S4800, Japan). The electric properties were real-time monitored by electrochemical workstation (Chenhua CHI 600E, China).

\section{Results and discussion}

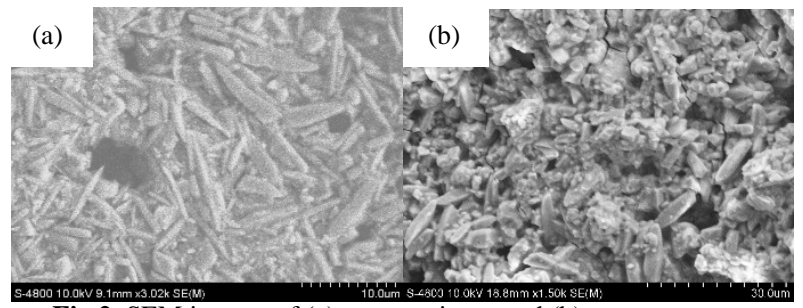

Fig 2. SEM images of (a) copper nitrate and (b) copper source.

Figure 2 shows SEM images of copper nitrate and copper salt source. Various shapes, such as irregular spheres, rods can be found. The size of copper particles is thus not uniform and ranges from hundreds nanometer to several micrometers. To confirm the component of copper salt, the
EDS was done and the spectrum was shown in Figure 3. The main element is copper, oxygen, carbon, and nitrogen.

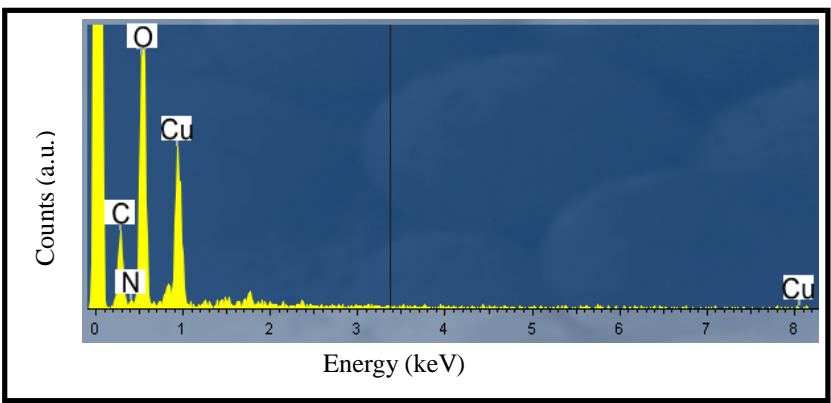

Fig 3. EDS spectrum of copper salt.

Figure 4 shows SEM images of reduced copper line with different laser powers The substrate is silica glass. Experimentally, the reaction cannot happen and the copper salt is only heated when laser power under $1 \mathrm{~W}$. The reduction threshold is $2 \mathrm{~W}$ and the obtained copper line width is about $200 \mu \mathrm{m}$. When the laser power increased to $3 \mathrm{~W}$, the copper line displays a good integrity. However, when the laser power is $5 \mathrm{~W}$ or higher, the laser begins to ablate the center of the copper line and even destroys the glass substrate as shown in figure 4 (c). The thickness of copper line is about $40 \mu \mathrm{m}$ shown in figure 5 . So the resistivity can be calculated by the equation: $r=R \cdot S / L$, where $r, R, S$ and $L$ are the resistivity, resistance, cross section area and length of copper line. The resistivity as a function of different laser powers is listed in table 1.

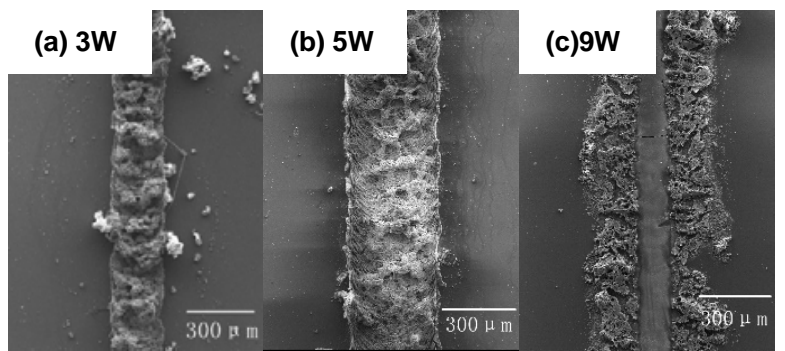

Fig 4. SEM images of copper line reduced by laser at the power of (a) $3 \mathrm{~W}$, (b) $5 \mathrm{~W}$ and (c) $9 \mathrm{~W}$.

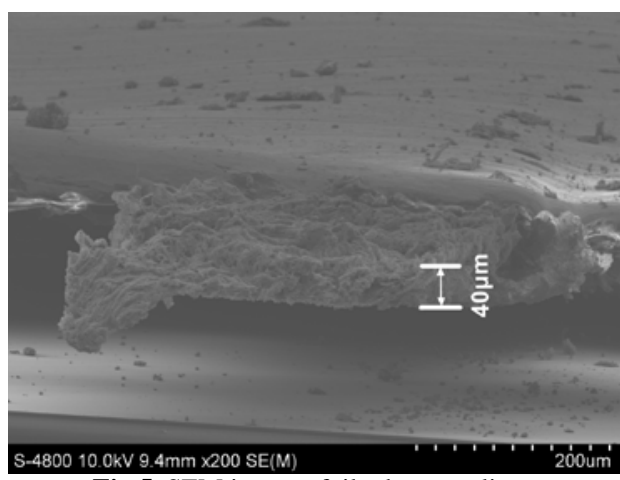

Fig 5. SEM image of tilted copper line.

When the temperature of the solution reaches $160-200{ }^{\circ} \mathrm{C}$, the ethylene glycol begins to be dehydrated and acetaldehyde reduces the copper salt to element copper [7, 10]. As the copper source decomposed at $250{ }^{\circ} \mathrm{C}$, the $\mathrm{NO}_{2}$ and $\mathrm{O}_{2}$ are created from nitrate. Besides, the formate can generate a 
large amount of CO gas at a low temperature $\left(101^{\circ} \mathrm{C}\right)$ [11]. This can enhance the reduction of $\mathrm{Cu}$ ion to $\mathrm{Cu}$ and inhibit re-oxide. To summarize, the reduction mechanism can be represented as Eq. 1

$$
2 \mathrm{HO}\left(\mathrm{CH}_{2}\right)_{2} \mathrm{OH}+\mathrm{Cu}^{2+} \longrightarrow \mathrm{Cu}+\mathrm{C}_{4} \mathrm{H}_{6} \mathrm{O}_{2}+4 \mathrm{H}^{+}+2 \mathrm{OH}^{-}
$$

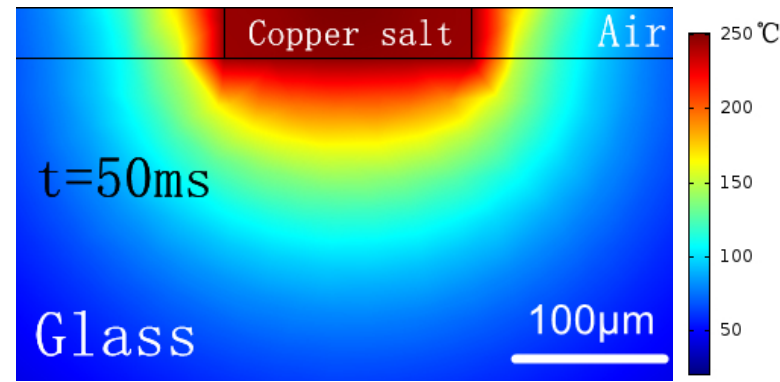

Fig 6. Cross-sectional temperature profiles at laser irradiation time $\mathrm{t}=0.05 \mathrm{~s}$

Figure 6 shows the simulation of cross-sectional temperature profiles after $50 \mathrm{~ms}$ laser irradiation. The laser beam is modeled as a heat source in the plane with a Gaussian profile and the radius is $75 \mu \mathrm{m}$. For the heat conduction/diffusion, we used a well-known equation that gives the time-dependent temperature decay $\mathrm{T}$ after the laser energy intensity $\mathrm{H}$ [12]:

$$
T=\left(\frac{H}{2 \rho C \sqrt{\pi \alpha}}\right) \frac{1}{\sqrt{\mathrm{t}}}
$$

Where $\rho$ is the density of the material, $\mathrm{C}$ is the specific heat and $\alpha$ is the thermal diffusivity of the material. Considering the experimental conditions, a conjugate heat transfer module with isothermal surroundings $(\mathrm{T}=300 \mathrm{~K}$ ) has been used in simulation. The model is surrounded by air and the underlying substrate is a typical glass with thermal conductivity of $1.4 \mathrm{~W} / \mathrm{m} \cdot \mathrm{K}$. The simulation results show that specimen temperature reaches $250{ }^{\circ} \mathrm{C}$ after $0.05 \mathrm{~s}$ irradiation. At such a temperature, the copper salt is decomposed and converted to $\mathrm{Cu}, \mathrm{NO}_{2}, \mathrm{H}_{2} \mathrm{O}$ and $\mathrm{O}_{2}$. That's the main reason that a large amount of gas is released under laser irradiation.

From Table 1, the optimal laser power is $3 \mathrm{~W}$ and the obtained resistivity is as low as $110 \mu \Omega \cdot \mathrm{cm}$. As a high laser power would partially ablate the reduced copper line and create many micro-cracks on the surface, the resistivity gradually increased with further increasing laser power. After patterned on PDMS, as shown in Figure 7, the resistivity increases to $240 \mu \Omega \cdot \mathrm{cm}$. When copper salt and ethylene glycol were being processed, $\mathrm{H}, \mathrm{C}, \mathrm{N}$ were released from salt as gas, such as $\mathrm{CO}_{2}, \mathrm{H}_{2} \mathrm{O}$ and so on [13]. Therefore, the copper line is porous and displays higher resistivity than bulk material. However, compared to some other group's results based on silver network, the resistivity what we obtained was much lower [5, 14]. In addition, as an elastomer, PDMS can be bent or stretched. After transferred on PDMS, flexible sensor based on copper electrodes can be constructed. From SEM images shown in Figure 7, the copper electrode was wrapped by PDMS, which would prevent the copper from oxidation.
Table 1. The resistivity of copper electrode reduced by laser.

\begin{tabular}{cc}
\hline Laser Power $(\mathrm{W})$ & Resistivity $(\mu \Omega \cdot \mathrm{cm})$ \\
\hline 2 & 420 \\
3 & 110 \\
5 & 115 \\
9 & 180 \\
\hline
\end{tabular}

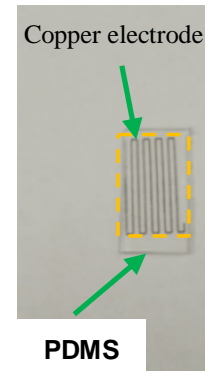

\section{(a)}

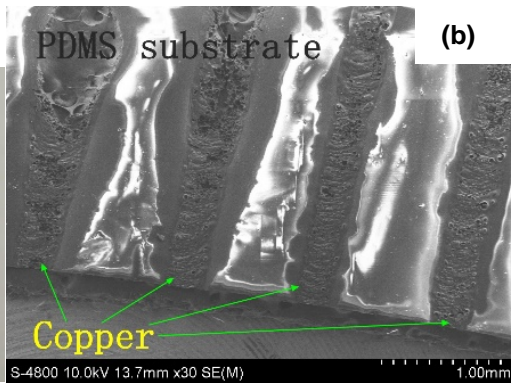

Fig 7. (a) Photograph and (b) SEM image of copper electrode patterned on PDMS.

To demonstrate the stretchability of our sensor, we measured resistance response $v s$. strain by a tensile tester. The results are showed in Figure 8. The range of strain is from $-10 \%$ (bending) 10\% (strain). The resistance with $10 \%$ strain is 30 times larger than the initial value, which illustrates our sensor is highly sensitive. We ascribe this excellent performance to the joined copper nanoparticles and porous structures, because the loose material is very sensitive to the deformation.

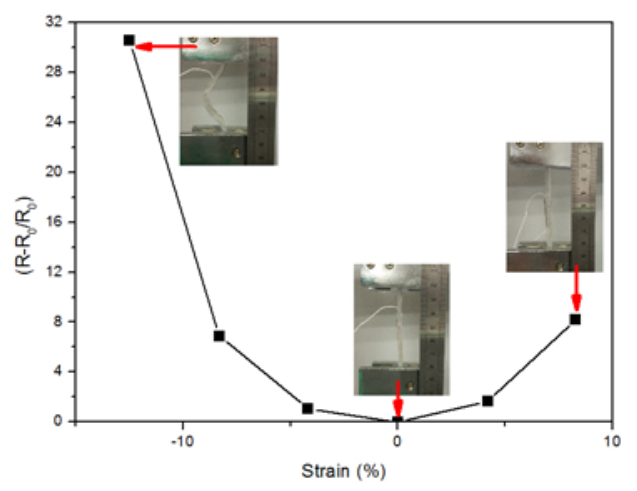

Fig 8. Resistance response of the sensor to strain.

PI (polyimide) is another important flexible substrate which can be used for human-machine interaction [15], health monitoring [16] and microheaters [7]. Figure 9 shows the copper electrodes and copper coil on a PI film. Because the copper pattern was sintered on PI film as laser processing, the copper pattern can be bent with PI film (Figure 9a). The insert of Figure 9b shows an antenna based on copper coil potentially for energy harvesting. The size of copper coil was $1 \mathrm{~cm} \times 1.5 \mathrm{~cm}$. The number of coils is 10 and the width of each copper line is about $300 \mu \mathrm{m}$. From Figure 9c, the reduced copper particles were sintered and joined together by laser $[17,18]$. Due to the sintering of copper particles, it is expected that the copper coils fabricated by laser reduction are more stable than isolated nanoparticles based on copper conductive ink. 


\section{(a)}

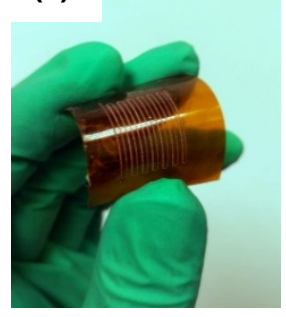

(b)

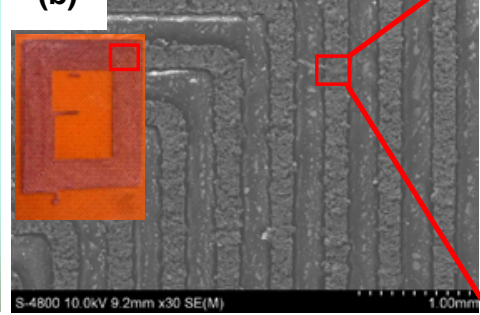

(c)

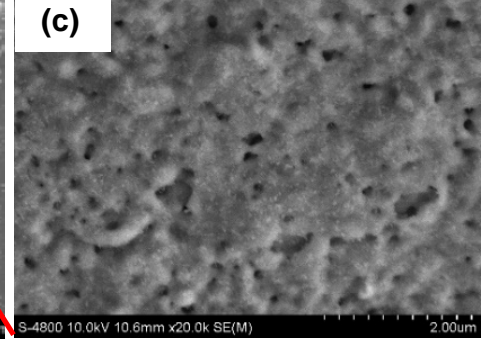

Fig 9. (a) Photograph of copper electrodes on PI film. (b) and (c) SEM images of copper coil on PI film.

As shown in Figure 10 (a) and (b), we fabricated a flexible sensor based on copper circuit, which used to control the lighting of LED. The LED will emit light after the electric circuit is pressured with $150 \mathrm{~g}$ weight on the top. At an unloaded state shown in the Figure 10 (a) and (c), the circuit is opened and there is no current to light LED. The deformation of sensor leads to short-cutting of circuits and the external power supply drives the lighting of LED through connected circuits as shown in Figure 10 (b) and (d).

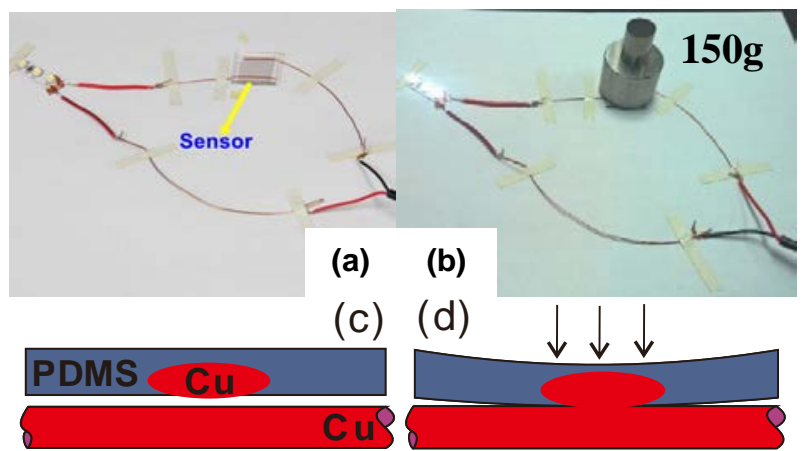

Fig 10. A pressure switch based on two-layer flexible copper circuits embedded in PDMS and the lighting control of LED. Photograph of senor with (a) and without wight (b). (c) and (d) show the working principle of the flexible switch.

\section{Conclusion}

We developed a new method to fabricate flexible sensors of copper circuits based on either PDMS or PI substrates by laser direct writing. Ethylene glycol was decomposed to aldehyde by laser heating and the copper was chemically reduced. The resistivity of copper circuit has been achieved on as low as $240 \mu \Omega \cdot \mathrm{cm}$ PDMS. We illustrated potential applications of the flexible devices such as copper antenna and pressure-switches. Therefore, this study is expected to contribute to advancing fabrication of flexible electronic devices for next-generation flexible electronics.

\section{Acknowledgement}

The study is partially supported by National Natural Science Foundation of China (51575016) and the Beijing Oversea High-Level Talent Project. A strategic research grant (KZ20141000500, B-type) of Beijing Natural Science Foundation P. R. China.

\section{References}

[1] M. Amjadi, A. Pichitpajongkit, S. Lee, S. Ryu and I. Park: ACS Nano, 8, (2014) 5154.

[2] S. Jung, J. H. Kim, J. Kim, S. Choi, J. Lee, I. Park, T. Hyeon and D. Kim: Adv. Mater., 26, (2014) 4825.

[3] X. Huang, Y. Liu, K. Chen, W. Shin, C. Lu, G. Kong, D. Patnaik, S. Lee, J. F. Cortes and J. A. Rogers: Small, 10, (2014) 3083

[4] A. Russo, B. Y. Ahn, J. J. Adams, E. B. Duoss, J. T. Bernhard and J. A. Lewis: Adv. Mater., 23, (2011) 3426

[5] J. Lee, S. Kim, J. Lee, D. Yang, B. C. Park, S. Ryu and I. Park: Nanoscale, 6, (2014) 11932.

[6] Y. Joo, J. Byun, N. Seong, J. Ha, H. Kim, S. Kim, T. Kim, H. Im, D. Kim and Y. Hong: Nanoscale, 7, (2015) 6208.

[7] Bongchul Kang, Seungyong Han, Jongsu Kim, Seunghwan Ko, and Minyang Yang: J. Phys. Chem. C, 115, (2011) 23664.

[8] R. Dharmadasa, M. Jha, D. A. Amos and T. Druffel: ACS Appl. Mater. Interfaces, 5, (2013) 13227.

[9] R. Li, A. Hu. D. Bridges, T. Zhang, K. D. Oakes, R. Peng, U. Tumuluri, Z. Wu and Z. Feng: Nanoscale, 7, (2015) 7368.

[10]F. Fievet, J. P. L agier, B. Blin: Solid State Ionics, 32/33, (1989) 198.

[11]B. Wang, T. Yoo, Y. Song, D. Lim and Y. Oh: ACS Appl. Mater. Interfaces, 5, (2013) 4113

[12]C. Zheng, A. Hu, K. D. Kihm, Q. Ma, R. Li, T. Chen and W. W. Duley: Small, 11, (2015) 3007

[13] G. L. Draper, R. Dharmadasa, M. E. Staats, B. W. Lavery and T. Druffel: ACS Appl. Mater. Interfaces, 7, (2015) 16478.

[14] Y. Wei, S. Chen, F. Li, Y. Lin, Y. Zhang and L. Liu: ACS Appl. Mater. Interfaces, 7, (2015) 14182.

[15]R. Rahimi, M. Ochoa, W. Yu and B. Ziaie: ACS Appl. Mater. Interfaces, 7, (2015) 4463.

[16] S. Luo, P. T. Hoang and T. Liu: Carbon, 96, (2016) 522.

[17] J. Yan, G. Zou, A. Hu and Y. N. Zhou: J. Mater. Chem., 21, (2011) 15981.

[18]A. Hu, J. Y. Guo, H. Alarifi, G. Patane, Y. Zhou, G. Compagnini and C. X. Xu: Appl. Phys. Lett., 97, (2010) 153117.

(Received: May 21, 2016, Accepted: August 31, 2016) 